\title{
Stress-associated factors in Mexican dentists
}

Blanca Elizabeth Pozos Radillo(a) Teresa Margarita Tórrez López ${ }^{(b)}$ María de Los Ángeles Aguilera Velasco(c)

Martin Acosta Fernández ${ }^{(\mathrm{d})}$ Guillermo Julian González Perez ${ }^{(\mathrm{e})}$

(a) Doctorate in Health Psychology, Research Professor; (b)Doctorate in Social Anthropology, Research Professor; (c)

Doctorate in Occupational Health, Research Professor - Public Health Department, University of Guadalajara, Mexico.

(d) Doctorate in Occupational Health, Research Professor; ${ }^{(e)}$ Doctorate in Social Medical Science, Research Professor

- Social Sciences Department, University of Guadalajara, Mexico.
Corresponding author: Blanca Elizabeth Pozos Radillo Paseo de los Virreyes 706 A-19

Virreyes Residencial

Zapopan - Jalisco - Mexico

C. P. 45110

E-mail: litaemx@yahoo.com.mx

Received for publication on Oct 23, 2007

Accepted for publication on May 12, 2008
Abstract: Dentistry is considered a stressful profession, since dentists are exposed to potential stressors during their practice. Therefore, the objective of this study was to identify chronic stress levels and their association with different risk factors among dentists working at public health institutions in Guadalajara, Mexico. The study was observational, crosssectional and one of association. The universe of this study was composed of 256 dentists that were obtained by means of a census technique. The instrument used for the analysis carried out in the year 2006 was the Stress Syndrome Inventory, performed with concurrent validation. Information was processed for the analysis, and chronic stress levels were identified with a bivariate analysis. Association strength was measured with OR, and confusion factors were controlled with a multivariate logistic analysis. Based on the obtained results, it was concluded that female dentists have a greater risk of developing a high chronic stress level with an adjusted OR of 1.84 , meaning that the risk for women is 1.84 times greater than that of men.

Descriptors: Dental staff; Occupational risk; Occupational medicine; Stress, psychological. 


\section{Introduction}

The historical origin of the concept of stress begins with Hans Selye's research in 1950 to differentiate stress conditions and the stimuli that cause them. ${ }^{1}$

Stress is a syndrome, as defined by Selye: An array of symptoms or signs that occur simultaneously, characterizing an illness, it is the mechanism that puts the entire system on alert with resistance and exhaustion in the face of situations, demands or environmental stimuli that threaten to imbalance the organism. ${ }^{2}$

Stress has gone from being an individual or personal problem to becoming a work and social prob$\mathrm{lem}^{3}$ that may affect individuals from all walks of life and ages, no matter what their gender, nationality, educational background or role, and has been specifically pointed out by health providers. ${ }^{4}$ Workrelated stress is estimated to affect at least a third of the workforce. Stress-related living factors have been proven to have influence on the development of innumerable diseases. ${ }^{5}$

The European Foundation estimates that lifestyle-related diseases explain at least half of all premature deaths. ${ }^{6}$ Dentists are exposed to stressors during dental practice that could put their health at risk; they may be classified as those existing in any of the dental procedures, and those related to office organization, patient-dentist relationship, and relationship with assistants. ${ }^{7}$ Several studies infer that dentists in general practice may experience greater physical and mental illness in comparison to other health professionals. ${ }^{8}$

The Swedish Dental Association claims that dentists live ten years less than the country's general population due to the occupational hazards they are exposed to, including psychological factors such as stress, emotional fatigue, tension, anguish and anxiety. ${ }^{9}$

Mention has been made in England that there is evidence suggesting that dentists currently suffer from a higher level of work-related stress, ${ }^{10}$ and according to the literature, little has been studied about stress among dentists since most studies have targeted other professions. Given its importance as an occupational hazard, the purpose of this study was to identify chronic stress levels (high, medium, low) of dentists, and the association of risk factors with high level chronic stress.

\section{Material and Methods}

The study was observational, cross-sectional and one of association. The criteria for sample inclusion were all dentists working at clinics and/or hospitals at public health institutions in Guadalajara, Jalisco, Mexico: Mexican Social Security Institute (IMSS), Ministry of Health Jalisco (SSJ), Family Services (DIF), Social Security Services Institute for Government Workers (ISSSTE), and the University of Guadalajara ( $U$ de $G$ ). They were chosen because they have a captive population. Dentists covering temporary posts, internships and those working at private practices were excluded due to a lack of reliable records of their location.

The Work Universe was composed of 256 dentists ( $\mathrm{n}=256)$ distributed as follows: 90 dentists at U de G, 68 dentists at IMSS, 44 at SSJ, 37 at DIF, and 17 at ISSSTE, as obtained by the census technique carried out in the year 2006.

The variables were: Chronic stress levels, gender, age, marital status, socioeconomic level, work seniority, work performed with an assistant, hours of work per day, other employment, family interaction time, and doing housework.

The tools used were: A questionnaire of the dentists' personal information and the Stress Symptom Inventory (SSI) questionnaire drafted and validated by Lipp, Guevara ${ }^{11}$ (1994).

Translated and adapted for Mexico by Dominguez, the SSI contains a list of 42 Psycho-physiological Symptoms characteristic of chronic stress, classified on the Likert Scale of 6 choices from "never" to "always". Chronic stress levels were governed for each institution by the average, and standard deviation (SD) was deemed high when it was between $(+2$ SD and +3 SD), medium level between $(-1$ SD and +

\footnotetext{
*Dominguez B. Draft report presented to the Research Department of the University Medical Center, UNAM, Mexico 1988, that presented the validation of SSI content, obtaining a Cronbach Alpha of .94, thus indicating an acceptable degree of reliability.
} 
$1 \mathrm{SD})$, and low between ( $-3 \mathrm{SD}$ and $-2 \mathrm{SD})$, thereby obtaining the levels present in each institution.

For the purpose of accurately identifying the chronic stress levels present among dentists at the Public Health Institutions, concurrent validation was performed for this study with the SSI (Stress Symptom Inventory) tool, which is the most appropriate validation for psychological tests applied to diagnosing existent status, and the Somatic Cognitive Anxiety Questionnaire (SCAQ) as the gold standard. This latter instrument was validated with a Cronbach Alpha greater than 0.80, indicating its reliability. ${ }^{12}$ The reason behind using this instrument is because anxiety is a manifestation of stress, thus indicating that stress increases to the same extent as does anxiety.

The concurrent validation of SSI in this study found a high positive correlation ratio as well as a meaningful $\mathrm{p}(\mathrm{p}<0.05)$, which indicates that the results are not random, and that we had a reliable instrument for measuring chronic stress levels that has validity and reliability qualities to discriminate between different levels of chronic stress.

The analysis was done in two stages. During stage one, information was processed by using rates and percentages identifying the stress levels present in this study's population.

During the second stage, a bivariate analysis was performed to identify risk factors, and the strength of association was assessed by OR. Risk factors were obtained by comparing extreme high levels with low levels of chronic stress after other comparisons were made between high levels and medium and low levels.

Confusion factors were controlled with a logistic multivariate analysis. The adjusted ratio showed a true association between the variables of this study and a high level of stress, thus avoiding the confusion effect of variable predictors that would muddle any evaluation of the results. There is a large amount of interrelated variables in this study, so an analysis of this kind was by all means necessary. The SPSS statistical program was used for statistical analysis.

The variables meeting the traits - age, gender, other employment and seniority - were considered confusion factors because they were risk factor for the studied disease, and were found to be associated with exposure and not to the consequence of exposure.

According to the Title Two of the General Health Act in Matters of Health Research for the Ministry of Health and Welfare in Jalisco, which addresses the ethical aspects of research on human beings, this research was classified in the following category: Minimal Exposure.

\section{Results}

Of the 256 Dentists, chronic high stress levels were found in $35(13.7 \%)$ of the dentists, medium level stress in $184(71.8 \%)$, and a low level of chronic stress in $37(14.5 \%)$ (Table 1$)$.

This Table shows a high tendency towards medium level chronic stress, with a rate of $71.8 \%$ of the affected population, as a red-flag situation, considering that the instrument measures stress with the presence of manifest symptoms.

As for age, $47.3 \%$ were between 40 and 49 years old, $47.3 \%$ had between 1 and 10 years of seniority, $57.8 \%$ worked from 4 to 6 hours a day, $72.7 \%$ did housework everyday, and $44.5 \%$ spent $50 \%$ of their time in interaction with their family. The prevailing gender was female, with $51.5 \%, 66.4 \%$ stated that they were married, $52.4 \%$ had a high socioeconomic level, $78.6 \%$ worked with an assistant, and $51.9 \%$ had another job.

The variable found to be associated with high level chronic stress according to the bivariate analy-

Table 1 - Chronic stress level rates among dentists distributed by institution in Guadalajara, Mexico (2006).

\begin{tabular}{c|r|r|r|r|r|r|r|r}
\hline & \multicolumn{7}{c}{ Chronic stress level } \\
\hline & \multicolumn{2}{|c|}{ High } & \multicolumn{2}{c}{ Medium } & \multicolumn{2}{|c|}{ Low } & \multicolumn{2}{c}{ Total } \\
\hline Institution & $\mathrm{n}$ & $\%$ & $\mathrm{n}$ & $\%$ & $\mathrm{n}$ & $\%$ & $\mathrm{n}$ & $\%$ \\
\hline IMSS & 9 & 3.5 & 55 & 21.5 & 4 & 1.6 & 68 & 26.6 \\
\hline SSJ & 9 & 3.5 & 27 & 10.6 & 8 & 3.1 & 44 & 17.2 \\
\hline DIF & 4 & 1.6 & 28 & 10.9 & 5 & 2 & 37 & 14.5 \\
\hline ISSSTE & 2 & 0.8 & 12 & 4.6 & 3 & 1.2 & 17 & 6.6 \\
\hline U de G & 11 & 4.3 & 62 & 24.2 & 17 & 6.6 & 90 & 35.1 \\
\hline Total & 35 & 13.7 & 184 & 71.8 & 37 & 14.5 & 256 & 100 \\
\hline
\end{tabular}

Direct sources. 


\begin{tabular}{|c|c|c|c|c|c|}
\hline \multirow{12}{*}{$\begin{array}{r}\text { Table } 2 \text { - } \\
\text { Sociodemographic factors } \\
\text { associated with high level } \\
\text { chronic stress. Results of } \\
\text { a bivariate analysis } \\
\text { in Guadalajara, } \\
\text { Mexico (2006). }\end{array}$} & Variables & N & OR & $\mathrm{Cl} 95 \%$ & $P$ \\
\hline & Sex: Female vs. Male & 23 & $* 4.53$ & $1.51-13.93$ & 0.002 \\
\hline & Socioeconomic level: Medium vs. High and Low & 18 & 1.25 & $0.45-3.49$ & 0.641 \\
\hline & Age: $40-49$ vs. $20-29,30-39$ and $50-59$ & 19 & $* 4.83$ & $1.55-15.5$ & 0.018 \\
\hline & Marital status: Single vs. Married Divorced and Widow & 11 & 1.67 & $0.55-5.14$ & 0.220 \\
\hline & Works with assistant: Yes vs. No & 19 & 2.81 & $0.96-8.35$ & 0.034 \\
\hline & Seniority: $21-30$ years vs. $1-10$ and $11-20$ & 7 & 3.36 & $0.71-17.9$ & 0.147 \\
\hline & Housework: Yes vs. No & 17 & 2.43 & $0.75-8.08$ & 0.096 \\
\hline & Hours worked during the day: $4 / 6$ vs. $7 / 9,10 / 12$ & 21 & 1.27 & $0.45-3.62$ & 0.610 \\
\hline & Other employment: Yes vs. No & 24 & 2.30 & $0.79-6.77$ & 0.136 \\
\hline & Family interaction time: $50 \%$ vs. $-30 \%,+75 \%$ & 17 & 1.24 & $0.44-3.49$ & 0.650 \\
\hline & Total & 35 & & & \\
\hline
\end{tabular}

sis using OR and its reliability intervals was: Female between the ages of 40 and 49 years (Table 2).

Only extreme values were used in the association analysis; i.e., chronic high level stress was taken as a risk by associating it with a low non-risk level due to not finding any statistically significant association when middle or low levels were analyzed as not posing risks.

No association was found for the variables of male gender, age, weight, height, number of children, marital status, socioeconomic level, work seniority, work with an assistant, hours worked daily, other employment, family interaction time, and housework.

Later, when applying a multivariate analysis where confusion factors were controlled, it was found that only the female gender maintained an association with adjusted OR of 1.84 and a CI of $95 \%$ of $1.10<$ OR $<3.06$, even after controlling for age variables between 40 and 49 , seniority between 21 and 30 years and not having other employment. Therefore, an association of the female gender with high level chronic stress was accepted (Table 3 ).

The results show that female dentists are particularly exposed to stress as a health hazard. This situation may be related to their profile, determined by working a double shift, underscoring the work process and housework that might be related to health and sickness. ${ }^{6}$ Socially imposed limits on a woman are not relaxed when she is integrated into
Table 3 - Association between chronic stress and age, female gender, other employment, and seniority. Results of a logistical regression model (multivariate analysis) in the metropolitan area of Guadalajara, Mexico (2006).

\begin{tabular}{|c|c|c|c|c|}
\hline Variables & $\begin{array}{l}\text { High Level } \\
\text { Subjects }\end{array}$ & $P$ & OR & $\mathrm{Cl}(95 \%)$ \\
\hline Age 40-49 & 20 & .919 & 0.96 & $0.45-2.07$ \\
\hline Female Gender & 23 & .019 & $* 1.84$ & $1.10-3.06$ \\
\hline $\begin{array}{l}\text { Not having } \\
\text { Another job }\end{array}$ & 24 & .099 & 1.91 & $0.89-4.12$ \\
\hline $\begin{array}{c}\text { Seniority of } 21 \\
\text { to } 30 \text { years }\end{array}$ & 7 & .113 & 2.24 & $0.83-6.08$ \\
\hline Constant & -2.836 & & & \\
\hline -2log Likelihood & 191.632 & & \multicolumn{2}{|c|}{$\begin{array}{l}\text { Nagelkerkê }-R^{2} \\
\quad=0.088\end{array}$} \\
\hline$X^{2}=12.637$ & & & \multicolumn{2}{|c|}{$P=.013$} \\
\hline
\end{tabular}

$\mathrm{OR}=$ Odds Ratio. $\mathrm{Cl}=$ Confidence Interval. ${ }^{*}$ Statistical significance at the level of 0.05 .

a paying job; rather, each dentist shapes them with her own strategies, with emphasis on a pathological stress profile.

\section{Discussion}

The purpose of this study to identify chronic stress levels (high, medium and low) found in dentists, and the association of risk factors with chronic high level stress was achieved.

Among the results obtained, the presence of medium level chronic stress stands out as a red-flag 
situation since at any given moment it could pass to high level chronic stress. This suggests that the time has come for dentists to carry out an intervention whose purpose would be to establish preventive measures to handle and control stress.

The bivariate analysis determined that the variables male gender, socioeconomic level, age 20-29 and 50-59, marital status, work with an assistant, seniority, housework, hours worked, other employment, and family interaction time did not show any association with high level chronic stress. The reason why these variables were chosen as possible stress-associated factors was that they have been referred to in other studies as important stressors, able to affect dentists' quality of life and their closest surroundings with consequences on their work performance. . $^{4,5,7}$

In the case of the variables female gender and age between 40 and 49 years, which the bivariate analysis associated significantly with high level chronic stress according to OR, these results coincide with those of other studies by some researchers who reported that women display greater stress levels. ${ }^{13}$ Others, on the contrary, report that the male gender is more susceptible to suffer from stress, and this may be due to the fact that the kind of stress being studied is situational or in the moment. ${ }^{14}$

As for age and stress, this study shows that the 40 to 49 year range has a significant association according to OR. This coincides with studies that show that older age groups show significantly more stress, and it may be due to the fact that older age brings about greater responsibilities and greater physical exhaustion. ${ }^{15}$ This could be translated into a greater risk of suffering high level chronic stress. Over $50 \%$ of the studied population was 40 years old or older.

Nevertheless, the multivariate analysis showed that only the female gender maintained a significant association with chronic stress even after controlling for confusion variables. This is consistent with other studies that show that women suffer more emotional exhaustion, ${ }^{3}$ depression, anguish crises, and participate in more suicide attempts (which could be triggered by chronic stress).

Regarding the search for association between chronic stress levels and their risk factors, we recommend controlling other factors that could influence modifications of the relation between stress, age and female gender. We also recommend that strategies be identified to confront stress, implementing preventive measures to moderate the negative effect of stress as a risk factor.

The limitation of this study was that it did not establish causality relations.

\section{Conclusion}

Proactive stress management is prevention aimed at developing behavioral changes that strengthen the capacity to cope with work pressure. ${ }^{8}$

Based on the results obtained, we conclude that female dentists are at a greater risk of developing high level chronic stress, almost twice as much as male dentists.

An accurate identification of stress and stressors in dental practice as well as greater understanding of the most common situations causing it may lead to reducing stress. It may eliminate its effects before it does harm to dentists' health and workplace safety.

Therefore, we recommend implementing preventive programs in health institutions aimed at managing and controlling dentists' stress with relaxation and visualization techniques among others.

\section{Acknowledgments}

This study was possible thanks to the participation of the public health institutions in Guadalajara, Mexico. The author and her collaborators thank the proofreaders of this article for their constructive suggestions as well as the participating dentists. 


\section{References}

1. Mello AA, Mello MF, Carpenter LL, Price LH. Update on stress and depression: the role of the hypothalamic-pituitaryadrenal (HPA) axis. Rev Bras Psiquiatr. [newspaper on-line] 2003 (cited 2007 Sep 14);25(4):231-8. Available from: http:// www.scielo.br/scielo.php?script $=$ sci_arttext $\sigma p i d=S 1516$ 44462003000400010むIng=en ónrm=iso.

2. Levitini M. El estrés: un abordaje múltiple. Buenos Aires: Editorial Policial; 2002. p. 7.

3. Fallon AE, Rozin P. Sex differences in perceptions of desirable body shape. J Abnorm Psychol. 1985 Feb;94(1):102-5.

4. Pando M, Bermúdez D, Aranda C, Pérez J, Flores E, Arellano G. Stress and Burnout in Health Providers in Guayana, Venezuela City. J Psicol y Salud. 2003 Jan-Jun;13(1):47-52.

5. Machado RV, Gauer GJC. Posttraumatic stress disorder and bipolar mood disorder. Rev Bras Psiquiatr. [newspaper online] 2003 [cited 2007 Sep 14]. Available from: http://www. scielo.br/scielo.php?script=sci_arttext 6 pid $=\$ 1516-444620$ 03000500013\&Ing=enむnrm=iso\&tng=pt.

6. Expert's reunions in Reus. Work stress affects more than 40 million European people. Health on line. [diário on-line] 2006 [citado 10 nov 2006]. Disponible en: http://www.consumer. es/web/es/salud/2006/11/10/157207.php.
7. Cureton T. Health and physical fitness tests of dentists (with implications). J Dent Med. 1961 Oct;16:211-23.

8. Enoch MD, Robert GJ. Psychiatric disorders in dental practice. Oxford: Boston Wright; 1994.

9. Kamin V. Fear, Stress and the Well Dental Office. Northwest Dent. 2006 Mar-Apr;85(2):10-1,13,15-8.

10. Branthwaite A, Ross A. Satisfaction and job stress in general practice. Fam Pract. 1988 Jun;5(2):83-93.

11. Lipp MEN, Guevara AJ de H. Validação empírica do Inventário de Sintomas de Stress (ISS). Estud Psicol. 1994;11(3):43-9.

12. Sandra S, Ivonne B. Validación del Cuestionario de Ansiedad Cognoscitiva - Somática [Tesis de Licenciatura]. Facultad de Psicología de la Universidad Nacional Autónoma de México; 1996.

13. Kelsey JL, Whittemore AS, Evans AS, Thompson WD. Methods in Observational Epidemiology. 2nd ed. New York: Oxford University Press; 1996.

14. González OR, Cuevas GL, Díaz De KM. La participación de las mujeres en la odontología (primera parte). Revista ADM 2000 Ene-Feb;57(1):19-22.

15. Meda L. Health and Stress in small factory workers from Guadalajara Jalisco [Doctoral thesis]. Jalisco: University Health Sciences Center at the University of Guadalajara; 1998. 